\title{
Molecular iodine-catalyzed one-pot synthesis of some new Hantzsch 1,4-dihydropyridines at ambient temperature
}

\author{
J. D. Akbari, S. D. Tala, M. F. Dhaduk, and H. S. Joshi* \\ Department of Chemistry, Saurashtra University, \\ Rajkot-360005, Gujarat, India \\ E-mail:drhsjoshi49@gmail.com,jdakbari@gmail.com
}

\begin{abstract}
An efficient and simple one-pot synthesis of some new symmetrical, unsymmetrical and Nsubstituted Hantzsch 1,4-dihydropyridines using molecular iodine as catalyst from an aldehyde, a 1,3-dicarbonyl compound and ammonium acetate / aromatic amine in ethanol is described. This new method has the advantage of good to excellent yields (80-95\%) and short reaction times (2.5-5 h) at ambient temperature.
\end{abstract}

Keywords: Hantzsch 1,4-dihydropyridines, molecular iodine, efficient and simple

\section{Introduction}

Hantzsch 1,4-dihydropyridines (1,4-DHPs) and their derivatives have gained great importance in the field of organic and medicinal chemistry since they display a fascinating array of pharmacological properties. ${ }^{1,5}$ The dihydropyridine skeleton is common in many drugs such as nifedipine, nicardipine, amlodipine and others, which are effective as cardiovascular agents and which are also used for the treatment of hypertension. ${ }^{6,7} 1,4$-DHPs have been explored for their calcium channel modulation ${ }^{8,9}$ and the heterocyclic rings are found in a variety of bioactive compounds such as vasodilator, bronchodilator, antiatherosclerotic, antitumor, antidiabetic, geroprotective and heptaprotective agents. ${ }^{10-13}$ They even possess neuroprotective platet antiaggregation activity. ${ }^{14}$ The tremendous drug activity of these compounds has attracted many chemists to synthesize these molecules.

More than 100 years ago the first 1,4-DHPs were synthesized by Hantzsch and Liebigs. ${ }^{15}$ The classical method involves a one-pot condensation of an aldehyde with 1,3-dicarbonyl compounds and ammonia either in acetic acid or in an refluxing alcohol for a longer time. ${ }^{16}$ Due to some disadvantages of this method, there are several efficient methods developed for the synthesis of 1,4-DHPs, which comprise the use of microwave, ${ }^{17-18}$ ionic liquid, ${ }^{20-21}$ high temperature in refluxing solvents, ${ }^{22-25}$ TMSCl-NaI $^{26}$ and metal triflates. ${ }^{27}$ However, the use of high 
temperatures, expensive metal precursors and longer reaction times limits these methods. Due to these problems, the development of an efficient and versatile method for the synthesis of 1,4DHPs is an active research area and there is room for further improvement towards mild reaction conditions and to improve the yields of the reaction.

Molecular iodine ${ }^{28-31}$ has attracted attention as an inexpensive, non toxic, readily available catalyst for various organic transformations to afford the corresponding products in excellent yields with high selectivity. It has been used as a mild Lewis acid in the dehydration of tertiary alcohols to alkenes, in the formation of ethers, as well as $\beta$-keto enol ethers, ${ }^{32-34}$ for esterification, ${ }^{35}$ transesterification, ${ }^{36}$ acetylation $^{29}$ and benzothiophene ${ }^{37}$ formation, but there are only a few reports about its use for the synthesis of 1,4 -DHPs. ${ }^{38,39}$ Therefore, we decided to synthesize some new symmetrical, unsymmetrical and N-substituted 1,4-DHPs promoted by a catalytic amount of iodine under ambient conditions with good to excellent yields. ${ }^{38}$

\section{Results and Discussion}

In the present work, based on a literature survey, ${ }^{38} 30 \mathrm{~mol} \%$ of catalyst was used throughout the experiments. One equiv. of different aromatic aldehydes 1 and ammonium acetate 3 with two equiv. of a 1,3-dicarbonyl compound 2 were stirred at room temperature in the presence of molecular iodine in a little amount of ethanol afforded the corresponding 1,4-DHPs 4a-f in 88$95 \%$ yield at room temperature (Scheme1). In a similar fashion, reaction of various aldehydes 1 reacted smoothly with 1,3-dicarbonyl compounds 2, methyl acetoacetate 5 and ammonium acetate 3 under these reaction conditions to give the corresponding unsymmetrical 1,4-DHPs derivatives 6a-f in $88-94 \%$ yield under ambient conditions (Scheme 2). No statistical mixture of symmetrical and unsymmetrical products has been obtained.

Next, we investigated the effect of iodine in the synthesis of N-substituted 1,4-DHPs. Aromatic aldehydes 1, such as 3-nitrobenzaldehyde, reacts with isopropyl acetoacetate 7 and aromatic amines 8 such as 4-methoxyaniline in the presence of iodine to afford the products 9a-d in moderate yields (81-84\%) as compared to $4 \mathrm{a}-\mathrm{f}$ and $6 \mathrm{a}-\mathrm{f}$ (Scheme 3 ). All the products were fully characterized by IR, ${ }^{1} \mathrm{H}$ NMR and mass spectral analysis.

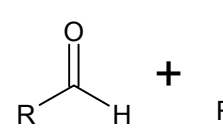

1<smiles>[R]C(=O)CC([R])=O</smiles>

2<smiles>[R]C(=O)C1=C([R])NC([R])=C(C([R])=O)C1[R]</smiles>

4a-f

Scheme 1. Synthesis of symmetrical 1,4-DHPs. 
<smiles>[R]C=O</smiles>

1<smiles>CC(=O)CC(=O)OC(C)C</smiles>

7<smiles>[R][CH][C@H](C)OC(=O)C1=C(C)N([R])C(C)=C(C(=O)OC(C)C)C1[R]</smiles>

9a-d

Scheme 2. Synthesis of unsymmetrical 1,4-DHPs.<smiles>[R]C(=O)[18F]</smiles>

1<smiles>CC(=O)CC(=O)OC(C)C</smiles>

7<smiles>N[Ga]</smiles>

8<smiles>[R]C1C(C(=O)OC(C)C)=C(C)N([R])C(C)=C1C(=O)OC(C)C</smiles>

9a-d

Scheme 3. Synthesis of N-substituted 1,4-DHPs.

Table 1. Synthesis of symmetrical, unsymmetrical, and N-substituted 1,4-DHPs using 0.3 mmol iodine as a catalyst through out the experiment at ambient temperature

\begin{tabular}{|c|c|c|c|c|c|c|c|c|}
\hline Entry & 1 & $\mathbf{R}$ & $\mathbf{R}_{1}$ & $\mathbf{R}_{2}$ & $\mathbf{R}^{\prime}$ & Product & Time & $\begin{array}{l}\text { Yield } \\
\%\end{array}$ \\
\hline 1 & $1 \mathrm{a}$ & & $-\mathrm{CH}\left(\mathrm{CH}_{3}\right)_{2}$ & $-\mathrm{OCH}_{3}$ & - & $4 a$ & $3 \mathrm{~h}$ & 95 \\
\hline 2 & $1 b$ & $\mathbf{O}_{2}$ & $-\mathrm{CH}\left(\mathrm{CH}_{3}\right)_{2}$ & $-\mathrm{OCH}_{3}$ & - & $4 b$ & $4 \mathrm{~h}$ & 89 \\
\hline 3 & $1 \mathrm{c}$ & & $-\mathrm{CH}\left(\mathrm{CH}_{3}\right)_{2}$ & $-\mathrm{OCH}_{3}$ & - & $4 c$ & $3 \mathrm{~h}$ & 92 \\
\hline 4 & $1 d$ & & $-\mathrm{CH}_{3}$ & $-\mathrm{OCH}\left(\mathrm{CH}_{3}\right)_{2}$ & - & $4 d$ & $2.5 \mathrm{~h}$ & 91 \\
\hline 5 & $1 b$ & & $-\mathrm{CH}_{3}$ & $-\mathrm{OCH}\left(\mathrm{CH}_{3}\right)_{2}$ & - & $4 \mathrm{e}$ & $3.5 \mathrm{~h}$ & 88 \\
\hline 6 & $1 \mathrm{c}$ & & $-\mathrm{CH}_{3}$ & $-\mathrm{OCH}\left(\mathrm{CH}_{3}\right)_{2}$ & - & $4 \mathrm{f}$ & $3 \mathrm{~h}$ & 93 \\
\hline 7 & 1a & & $-\mathrm{CH}\left(\mathrm{CH}_{3}\right)_{2}$ & $-\mathrm{OCH}_{3}$ & - & $6 a$ & $3 \mathrm{~h}$ & 94 \\
\hline 8 & $1 b$ & $\mathbf{o}_{z^{\prime}}$ & $-\mathrm{CH}\left(\mathrm{CH}_{3}\right)_{2}$ & $-\mathrm{OCH}_{3}$ & - & $6 b$ & $4 \mathrm{~h}$ & 89 \\
\hline
\end{tabular}


Table 1. Continued

\begin{tabular}{|c|c|c|c|c|c|c|c|c|}
\hline Entry & 1 & $\mathbf{R}$ & R1 & $\mathbf{R 2}$ & $\mathbf{R}^{\prime}$ & Product & Time & $\begin{array}{l}\text { Yield } \\
\%\end{array}$ \\
\hline 9 & $1 \mathrm{c}$ & & $-\mathrm{CH}\left(\mathrm{CH}_{3}\right)_{2}$ & $-\mathrm{OCH}_{3}$ & - & $6 c$ & $3.5 \mathrm{~h}$ & 91 \\
\hline 11 & $1 \mathrm{a}$ & & $-\mathrm{CH}_{3}$ & $-\mathrm{OCH}\left(\mathrm{CH}_{3}\right)_{2}$ & - & $6 \mathrm{~d}$ & $3.5 \mathrm{~h}$ & 89 \\
\hline 11 & $1 \mathrm{~b}$ & & $-\mathrm{CH}_{3}$ & $-\mathrm{OCH}\left(\mathrm{CH}_{3}\right)_{2}$ & - & $6 e$ & $4 \mathrm{~h}$ & 88 \\
\hline 12 & $1 \mathrm{c}$ & & $-\mathrm{CH}_{3}$ & $-\mathrm{OCH}\left(\mathrm{CH}_{3}\right)_{2}$ & - & $6 f$ & $3.5 \mathrm{~h}$ & 91 \\
\hline 12 & $1 b$ & & - & - & & $9 a$ & $4 \mathrm{~h}$ & 81 \\
\hline 14 & $1 \mathrm{~b}$ & & - & - & & $9 b$ & $4.5 \mathrm{~h}$ & 83 \\
\hline 15 & $1 \mathrm{c}$ & & - & - & & $9 c$ & $5 \mathrm{~h}$ & 83 \\
\hline 16 & $1 \mathrm{c}$ & & - & - & & $9 d$ & $5 \mathrm{~h}$ & 84 \\
\hline
\end{tabular}

In conclusion, in this article an efficient and convenient process has been used to prepare various symmetrical, unsymmetrical and N-substituted Hantzsch 1,4-dihydropyridines from the reaction of different araldehydes, 1,3-dicarbonyl compounds, and ammonium acetate / aryl amines in the presence of a catalytic amount of iodine at ambient temperature. The present methodology offers very attractive features such as shorter reaction times, milder conditions, simplicity of the reaction, good to excellent product yields and commercially available iodine as a powerful catalyst for the synthesis of 1,4-DHPs.

\section{Experimental Section}

General Procedures. Melting points were estimated in open capillaries and are uncorrected. IR spectra were recorded on $\mathrm{KBr}$ discs, using FTIR-8400 spectrophotometer. ${ }^{1} \mathrm{H}$ NMR spectra were taken on a Bruker AVANCE II 400 spectrometer in $\mathrm{CDCl}_{3} / \mathrm{DMSO} \mathrm{d}_{6}$. Chemical shifts $\delta$ are given in ppm relative to TMS. Mass spectra were determined using direct inlet probe on a 
GCMS-QP2010 mass spectrometer (Shimadzu). Elemental analyses were performed on a Carlo Erba EA 1108 elemental analyzer at SAIF, CDRI Lucknow. Reactions were monitored on Merck aluminium thin layer chromatography (TLC, UV 254nm) plates. Visualization was accomplished either on UV chamber or in iodine vapour. Column chromatography was carried out on silica gel (60-120 mesh, Merck chemicals).

\section{General procedure for the synthesis of symmetrical 1,4-dihydropyridines (4a-f)}

A mixture of aromatic aldehydes 1 (5 mmol), 1,3-dicarbonyl compounds 2 (10 mmol), ammonium acetate $3(0.358 \mathrm{~g}, 5 \mathrm{mmol})$ and iodine $(0.38 \mathrm{~g}, 1.5 \mathrm{mmol})$ was charged in a $25 \mathrm{ml}$ round bottom flask containing $2 \mathrm{ml}$ of ethanol. The reaction mixture was then stirred at room temperature until the reaction was completed (2.5-4 $\mathrm{hr}$ monitored by TLC). The reaction mixture was treated with aq $\mathrm{Na}_{2} \mathrm{~S}_{2} \mathrm{O}_{3}$ solution, extracted into ethyl acetate $(2 \times 20 \mathrm{ml})$. The solvent was removed in vacuo and the resulting crude product (88-95\%) was recrystallized from ethanol to give the pure compounds $4 \mathrm{a}-\mathrm{f}$. The reaction time and yields are depicted in Table 1.

Dimethyl-2,6-diisopropyl-4-phenyl-1,4-dihydropyridine-3,5-dicarboxylate (4a). Yellow solid; mp 133-134 ${ }^{\circ} \mathrm{C}$; IR (KBr): $v_{\max }=3300(-\mathrm{NH}), 1670(-\mathrm{C}=\mathrm{O}), 1589(-\mathrm{C}=\mathrm{C}), 1569,1485,1429$, 1225, 1170, 1089, 1012, $837 \mathrm{~cm}^{-1} ;{ }^{1} \mathrm{H} \mathrm{NMR}\left(400 \mathrm{MHz}, \mathrm{CDCl}_{3}\right), \delta=1.25\left(\mathrm{~d}, 12 \mathrm{H},-\mathrm{CH}_{3}\right), 3.62(\mathrm{~s}$, $\left.6 \mathrm{H},-\mathrm{OCH}_{3}\right), 4.21(\mathrm{~m}, 2 \mathrm{H},-\mathrm{CH}), 5.12(\mathrm{~s}, 1 \mathrm{H},-\mathrm{CH}), 6.39$ (s, 1H, -NH), 7.32-8.05 (m, 5H, Ar-H); MS (EI): $m / z$ 357. Anal. Calcd. for $\mathrm{C}_{21} \mathrm{H}_{27} \mathrm{NO}_{4}$ : C, 70.56; H, 7.61; N, 3.92\%. Found: C, 70.41; H, $7.57 ; \mathrm{N}, 3.88 \%$.

Dimethyl-2,6-diisopropyl-4-(3-nitrophenyl)-1,4-dihydropyridine-3,5-dicarboxylate

(4b). Yellow solid; $\mathrm{mp}$ 138-140 ${ }^{\circ} \mathrm{C}$; IR (KBr): $v_{\max }=3305(-\mathrm{NH}), 1670(-\mathrm{C}=\mathrm{O}), 1628(-\mathrm{C}=\mathrm{C}), 1587$, $1569,1485,1427,1232,1180,1091 \mathrm{~cm}^{-1} ;{ }^{1} \mathrm{H} \mathrm{NMR}\left(400 \mathrm{MHz}, \mathrm{CDCl}_{3}\right), \delta=1.21\left(\mathrm{~d}, 12 \mathrm{H},-\mathrm{CH}_{3}\right)$, $3.67\left(\mathrm{~s}, 6 \mathrm{H},-\mathrm{OCH}_{3}\right), 4.20(\mathrm{~m}, 2 \mathrm{H},-\mathrm{CH}), 5.13(\mathrm{~s}, 1 \mathrm{H},-\mathrm{CH}), 6.31(\mathrm{~s}, 1 \mathrm{H},-\mathrm{NH}), 7.28-8.01(\mathrm{~m}, 4 \mathrm{H}$, $\mathrm{Ar}-\mathrm{H}) ;{ }^{13} \mathrm{C}$ NMR (400 MHz, $\mathrm{CDCl}_{3}$ ), 20.35, 20.53, 27.99, 39.39, 51.28, 101.44, 121.38, 122.36, 128.91, 133.77, 148.36, 149.44, 153.97, 167.15; MS (EI): $m / z$ 402. Anal. Calcd. for $\mathrm{C}_{21} \mathrm{H}_{26} \mathrm{~N}_{2} \mathrm{O}_{6}$ : C, 62.67; H, 6.51; N, 6.96\%. Found: C, 62.54; H, 6.44; N, 6.88\%.

Dimethyl-2,6-diisopropyl-4-(4-methoxyphenyl)-1,4-dihydropyridine-3,5-dicarboxylate (4c). Pale yellow solid; mp 129-130 ${ }^{\circ} \mathrm{C}$; IR (KBr): $v_{\max }=3305(-\mathrm{NH}), 1672(-\mathrm{C}=\mathrm{O}), 1628(-\mathrm{C}=\mathrm{C})$, $1587,1548,1485,1427,1232,1180,1091,904,776 \mathrm{~cm}^{-1} ;{ }^{1} \mathrm{H}$ NMR $\left(400 \mathrm{MHz}, \mathrm{CDCl}_{3}\right), \delta=1.24$ $\left(\mathrm{d}, 12 \mathrm{H},-\mathrm{CH}_{3}\right), 3.58\left(\mathrm{~s}, 6 \mathrm{H},-\mathrm{OCH}_{3}\right), 3.63\left(\mathrm{~s}, 6 \mathrm{H},-\mathrm{OCH}_{3}\right), 4.22(\mathrm{~m}, 2 \mathrm{H},-\mathrm{CH}), 5.18(\mathrm{~s}, 1 \mathrm{H},-\mathrm{CH})$, 6.29 (s, 1H, -NH), 7.30-8.01 (m, 4H, Ar-H); MS (EI): $m / z$ 387. Anal. Calcd. for $\mathrm{C}_{22} \mathrm{H}_{29} \mathrm{NO}_{5}$ : C, 68.20 ; H, 7.54; N, 3.61\%. Found: C, 68.01; H, 7.45; N, 3.42\%.

Diisopropyl-2,6-dimethyl-4-(3,4-dimethoxyphenyl)-1,4-dihydropyridine-3,5-dicarboxylate (4d). Pale yellow solid; mp 118-119 ${ }^{\circ} \mathrm{C}$; IR (KBr): $v_{\max }=3315(-\mathrm{NH}), 1687(-\mathrm{C}=\mathrm{O}), 1485,1429$, 1321, 1230, 1170, 1098, $1041 \mathrm{~cm}^{-1} ;{ }^{1} \mathrm{H}$ NMR (400 MHz, $\left.\mathrm{CDCl}_{3}\right), \delta=1.15\left(\mathrm{~d}, 6 \mathrm{H},-\mathrm{CH}_{3}\right), 1.25$ $\left(\mathrm{d}, 6 \mathrm{H},-\mathrm{CH}_{3}\right), 2.31\left(\mathrm{~s}, 6 \mathrm{H},-\mathrm{CH}_{3}\right), 3.81\left(\mathrm{~s}, 3 \mathrm{H},-\mathrm{OCH}_{3}\right), 3.83\left(\mathrm{~s}, 3 \mathrm{H},-\mathrm{OCH}_{3}\right), 4.95(\mathrm{~m}, 2 \mathrm{H},-\mathrm{CH})$, $5.00(\mathrm{~s}, 1 \mathrm{H},-\mathrm{CH}), 5.80(\mathrm{~s}, 1 \mathrm{H},-\mathrm{NH}), 6.70-6.88$ (m, 3H, Ar-H); MS (EI): $m / z$ 417. Anal. Calcd. for $\mathrm{C}_{23} \mathrm{H}_{31} \mathrm{~N}_{2} \mathrm{O}_{6}$ : C, 66.17; H, 7.48; N, 3.35\%. Found: C, 66.11; H, 7.44; N, 3.21\%. 
Diisopropyl-2,6-dimethyl-4-(3-nitrophenyl)-1,4-dihydropyridine-3,5-dicarboxylate

(4e). Yellow solid; mp 123-125 ${ }^{\circ} \mathrm{C}$; IR (KBr): $v_{\max }=3342(-\mathrm{NH}), 1627(-\mathrm{C}=\mathrm{O}), 1587(-\mathrm{C}=\mathrm{C}), 1569$, 1481, 1423, 1232, 1180, $1091 \mathrm{~cm}^{-1} ;{ }^{1} \mathrm{H}$ NMR $\left(400 \mathrm{MHz}, \mathrm{CDCl}_{3}\right), \delta=1.11\left(\mathrm{~d}, 6 \mathrm{H},-\mathrm{CH}_{3}\right), 1.24$ (d, 6H, $\left.-\mathrm{CH}_{3}\right), 2.34$ (s, 6H, - $\left.\mathrm{CH}_{3}\right), 4.95(\mathrm{~m}, 2 \mathrm{H},-\mathrm{CH}), 5.07(\mathrm{~s}, 1 \mathrm{H},-\mathrm{CH}), 6.23(\mathrm{~s}, 1 \mathrm{H},-\mathrm{NH}), 7.29-$ 8.14 (m, 4H, Ar-H); MS (EI): $m / z$ 402. Anal. Calcd. for $\mathrm{C}_{21} \mathrm{H}_{26} \mathrm{~N}_{2} \mathrm{O}_{6}: \mathrm{C}, 62.67 ; \mathrm{H}, 6.51 ; \mathrm{N}$, $6.96 \%$. Found: C, 62.54; H, 6.49; N, 6.79\%.

Diisopropyl-2,6-dimethyl-4-(4-methoxyphenyl)-1,4-dihydropyridine-3,5-dicarboxylate (4f). Pale yellow solid; mp 132-133 ${ }^{\circ} \mathrm{C}$; IR (KBr): $v_{\max }=3436(-\mathrm{NH}), 1631(-\mathrm{C}=\mathrm{O}), 1587(-\mathrm{C}=\mathrm{C})$, $1566,1481,1423,1228,1178,1089,921,773 \mathrm{~cm}^{-1} ;{ }^{1} \mathrm{H}$ NMR $\left(400 \mathrm{MHz}, \mathrm{CDCl}_{3}\right), \delta=1.13(\mathrm{~d}$, $\left.6 \mathrm{H},-\mathrm{CH}_{3}\right), 1.24\left(\mathrm{~d}, 6 \mathrm{H},-\mathrm{CH}_{3}\right), 2.28\left(\mathrm{~s}, 6 \mathrm{H},-\mathrm{CH}_{3}\right), 3.74\left(\mathrm{~s}, 3 \mathrm{H},-\mathrm{OCH}_{3}\right), 4.93(\mathrm{~m}, 2 \mathrm{H},-\mathrm{CH}), 4.98$ (s, 1H, -CH), $5.85(\mathrm{~m}, 1 \mathrm{H},-\mathrm{NH}), 6.72-7.26(\mathrm{~m}, 4 \mathrm{H}, \mathrm{Ar}-\mathrm{H})$; MS (EI): $\mathrm{m} / z$ 387. Anal. Calcd. for $\mathrm{C}_{22} \mathrm{H}_{29} \mathrm{NO}_{5}: \mathrm{C}, 62.20 ; \mathrm{H}, 7.54 ; \mathrm{N}, 3.61 \%$. Found: $\mathrm{C}, 62.01 ; \mathrm{H}, 6.45 ; \mathrm{N}, 3.48 \%$.

\section{General procedure for the synthesis of unsymmetrical 1,4-dihydropyridines (6a-f)}

A mixture of aryl aldehydes 1 (5 mmol), 1,3-dicarbonyl compound $2(5 \mathrm{mmol})$, methyl acetoacetate $5(1.079 \mathrm{ml}, 5 \mathrm{mmol})$, ammonium acetate $3(0.358 \mathrm{~g}, 5 \mathrm{mmol})$ and iodine $(0.38 \mathrm{~g}$, $1.5 \mathrm{mmol})$ was charged in a $25 \mathrm{ml}$ round bottom flask containing ethanol $(2 \mathrm{ml})$. The reaction mixture was then stirred at room temperature until the reaction was completed (3-4 hr monitored by TLC). The reaction mixture was treated with aq $\mathrm{Na}_{2} \mathrm{~S}_{2} \mathrm{O}_{3}$ solution, extracted into ethyl acetate $(2 \times 20 \mathrm{ml})$. The solvent was removed in vacuo and the resulting crude product (88-94\%) was recrystallized from the ethanol to give the pure compounds $6 \mathrm{a}-\mathrm{f}$. The reaction time and yields are depicted in Table 1.

Dimethyl-2-isopropyl-6-methyl-4-phenyl-1,4-dihydropyridine-3,5-dicarboxylate (6a). Light yellow solid; $\mathrm{mp} 142-143{ }^{\circ} \mathrm{C}$; IR $(\mathrm{KBr}): v_{\max }=3344(-\mathrm{NH}), 1651(-\mathrm{C}=\mathrm{O}), 1622(-\mathrm{C}=\mathrm{C}), 1581$, $1548,1488,1442,1326,1332,1180,1089 \mathrm{~cm}^{-1} ;{ }^{1} \mathrm{H}$ NMR (400 MHz, DMSO d 6 ), $\delta=1.09$ (d, $\left.3 \mathrm{H},-\mathrm{CH}_{3}\right), 1.17\left(\mathrm{~d}, 3 \mathrm{H},-\mathrm{CH}_{3}\right), 1.77(\mathrm{~m}, 1 \mathrm{H},-\mathrm{CH}), 2.35\left(\mathrm{~s}, 3 \mathrm{H},-\mathrm{CH}_{3}\right), 3.22\left(\mathrm{~s}, 3 \mathrm{H},-\mathrm{OCH}_{3}\right), 3.26$ (s, 3H, $\left.-\mathrm{OCH}_{3}\right), 4.02(\mathrm{~s}, 1 \mathrm{H},-\mathrm{CH}), 5.05(\mathrm{~s}, 1 \mathrm{H},-\mathrm{NH}), 7.30-8.12(\mathrm{~m}, 5 \mathrm{H}, \mathrm{Ar}-\mathrm{H}) ; \mathrm{MS}(\mathrm{EI}): \mathrm{m} / \mathrm{z}$ 329. Anal. Calcd. for $\mathrm{C}_{19} \mathrm{H}_{23} \mathrm{NO}_{4}$ : C, 69.28; H, 7.04; N, 4.25\%. Found: $\mathrm{C}, 69.14 ; \mathrm{H}, 6.94 ; \mathrm{N}$, $4.12 \%$.

Dimethyl-2-isopropyl-6-methyl-4-(3-nitrophenyl)-1,4-dihyd ropyridine-3,5-dicarboxylate (6b). Yellow solid; mp 139-140 ${ }^{\circ} \mathrm{C}$; IR (KBr): $v_{\max }=3446,3321(-\mathrm{NH}), 1631(-\mathrm{C}=\mathrm{O}), 1587$ $(-\mathrm{C}=\mathrm{C}), 1566,1489,1437,1321,1232,1182 \mathrm{~cm}^{-1} ;{ }^{1} \mathrm{H}$ NMR $\left(400 \mathrm{MHz}, \mathrm{DMSO} \mathrm{d}_{6}\right), \delta=0.97(\mathrm{~d}$, $\left.3 \mathrm{H},-\mathrm{CH}_{3}\right), 1.10\left(\mathrm{~d}, 3 \mathrm{H},-\mathrm{CH}_{3}\right), 1.79(\mathrm{~m}, 1 \mathrm{H},-\mathrm{CH}), 2.34\left(\mathrm{~s}, 3 \mathrm{H},-\mathrm{CH}_{3}\right), 3.23\left(\mathrm{~s}, 3 \mathrm{H},-\mathrm{OCH}_{3}\right), 3.46$ (s, 3H, - $\left.\mathrm{OCH}_{3}\right), 4.04(\mathrm{~s}, 1 \mathrm{H},-\mathrm{CH}), 4.99(\mathrm{~s}, 1 \mathrm{H},-\mathrm{NH}), 7.37-8.03(\mathrm{~m}, 4 \mathrm{H}, \mathrm{Ar}-\mathrm{H}) ;{ }^{13} \mathrm{C}$ NMR $(400$ $\left.\mathrm{MHz}, \mathrm{CDCl}_{3}\right), 15.32,16.66,20.25,35.92,41.89,49.34,51.31,53.77,81.23,95.87,120.66$, 121.18, 128.52, 133.35, 147.47, 151.44, 167.08, 172.65; MS (EI): $\mathrm{m} / \mathrm{z} 374$. Anal. Calcd. for $\mathrm{C}_{19} \mathrm{H}_{22} \mathrm{~N}_{2} \mathrm{O}_{6}: \mathrm{C}, 60.95 ; \mathrm{H}, 5.92 ; \mathrm{N}, 7.48 \%$. Found: $\mathrm{C}, 60.84 ; \mathrm{H}, 6.79 ; \mathrm{N}, 7.36 \%$.

Dimethyl-2-isopropyl-6-methyl-4-(4-methoxyphenyl)-1,4-dihydropyridine-3,5-dicarboxylate (6c). Pale yellow solid; $\mathrm{mp} 129-130{ }^{\circ} \mathrm{C}$; IR (KBr): $v_{\max }=3301(-\mathrm{NH}), 1662 \quad(-\mathrm{C}=\mathrm{O})$, $1589(-\mathrm{C}=\mathrm{C}), 1477,1787,1319,1230,1182,1089,1024,700 \mathrm{~cm}^{-1} ;{ }^{1} \mathrm{H}$ NMR $(400 \mathrm{MHz}$, DMSO 
$\left.\mathrm{d}_{6}\right), \delta=1.13\left(\mathrm{~d}, 3 \mathrm{H},-\mathrm{CH}_{3}\right), 1.22\left(\mathrm{~d}, 3 \mathrm{H},-\mathrm{CH}_{3}\right), 1.81(\mathrm{~m}, 1 \mathrm{H},-\mathrm{CH}), 2.31\left(\mathrm{~s}, 3 \mathrm{H},-\mathrm{CH}_{3}\right), 3.22(\mathrm{~s}$, $\left.3 \mathrm{H},-\mathrm{CH}_{3}\right), 3.28\left(\mathrm{~s}, 3 \mathrm{H},-\mathrm{OCH}_{3}\right), 3.44\left(\mathrm{~s}, 3 \mathrm{H},-\mathrm{OCH}_{3}\right), 4.07(\mathrm{~s}, 1 \mathrm{H},-\mathrm{CH}), 5.08(\mathrm{~s}, 1 \mathrm{H},-\mathrm{NH}), 6.75-$ 7.32 (m, 4H, Ar-H); MS (EI): $m / z$ 359. Anal. Calcd. for $\mathrm{C}_{20} \mathrm{H}_{25} \mathrm{NO}_{5}: \mathrm{C}, 66.83 ; \mathrm{H}, 7.01 ; \mathrm{N}, 3.90 \%$. Found: C, 66.71; H, 6.88; N, 3.87\%.

Isopropyl methyl 2,6-dimethyl-4-phenyl-1,4-dihydropyridine-3,5-dicarboxylate (6d). Yellow solid; mp 124-126 ${ }^{\circ} \mathrm{C}$; IR (KBr): $v_{\max }=3352(-\mathrm{NH}), 1654(-\mathrm{C}=\mathrm{O}), 1614(-\mathrm{C}=\mathrm{C}), 1589$, 1491, 1444, 1328, 1226, 1161, $1026 \mathrm{~cm}^{-1} ;{ }^{1} \mathrm{H}$ NMR (400 MHz, DMSO d $), \delta=1.18(\mathrm{~d}, 3 \mathrm{H}$, $\left.-\mathrm{CH}_{3}\right), 1.23\left(\mathrm{~d}, 3 \mathrm{H},-\mathrm{CH}_{3}\right), 2.34\left(\mathrm{~s}, 3 \mathrm{H},-\mathrm{CH}_{3}\right), 2.37\left(\mathrm{~s}, 3 \mathrm{H},-\mathrm{CH}_{3}\right), 3.72\left(\mathrm{~s}, 3 \mathrm{H},-\mathrm{OCH}_{3}\right), 4.32(\mathrm{~m}$, 1H, -CH), 4.43 (s, 1H, -CH), 5.25 (s, 1H, -NH), 7.06-8.32 (m, 5H, Ar-H); MS (EI): m/z 329. Anal. Calcd. for $\mathrm{C}_{19} \mathrm{H}_{23} \mathrm{NO}_{4}$ : C, 69.28; H, 7.04; N, 4.25\%. Found: C, 69.13; H, 6.91; N, 4.10\%.

Isopropyl methyl 2,6-dimethyl-4-(3-nitrophenyl)-1,4-dihydropyridine-3,5-dicarboxylate (6e). Yellow solid; mp 137-138 ${ }^{\circ} \mathrm{C}$; IR (KBr): $v_{\max }=3336(-\mathrm{NH}), 1668(-\mathrm{C}=\mathrm{O}), 1608(-\mathrm{C}=\mathrm{C})$, 1581, 1489, 1446, 1227, 1165, 1047, 1012, $756 \mathrm{~cm}^{-1} ;{ }^{1} \mathrm{H}$ NMR (400 MHz, DMSO d 6 ), $\delta=1.19$ $\left(\mathrm{d}, 3 \mathrm{H},-\mathrm{CH}_{3}\right), 1.25\left(\mathrm{~d}, 3 \mathrm{H},-\mathrm{CH}_{3}\right), 2.39\left(\mathrm{~s}, 3 \mathrm{H},-\mathrm{CH}_{3}\right), 2.40\left(\mathrm{~s}, 3 \mathrm{H},-\mathrm{CH}_{3}\right), 3.75\left(\mathrm{~s}, 3 \mathrm{H},-\mathrm{OCH}_{3}\right)$, 4.35 (m, 1H, -CH), 4.44 (s, 1H, -CH), 5.31 (s, 1H, -NH), 7.29-8.10 (m, 4H, Ar-H); MS (EI): $m / z$ 374. Anal. Calcd. for $\mathrm{C}_{19} \mathrm{H}_{22} \mathrm{~N}_{2} \mathrm{O}_{6}$ : C, 60.95; H, 5.92; N, 7.48\%. Found: C, 60.86; H, 6.78; N, $7.39 \%$.

Isopropyl methyl 2,6-dimethyl-4-(4-methoxyphenyl)-1,4-dihydropyridine-3,5-dicarboxylate (6f). Pale yellow solid; mp 121-122 ${ }^{\circ} \mathrm{C}$; IR (KBr): $v_{\max }=3322(-\mathrm{NH}), 1676(-\mathrm{C}=\mathrm{O}), 1628$ $(-\mathrm{C}=\mathrm{C}), 1584,1552,1478,1436,1322,1228,1179,1079 \mathrm{~cm}^{-1} ;{ }^{1} \mathrm{H}$ NMR $\left(400 \mathrm{MHz}, \mathrm{DMSO} \mathrm{d}_{6}\right)$, $\delta=1.12\left(\mathrm{~d}, 3 \mathrm{H},-\mathrm{CH}_{3}\right), 1.17\left(\mathrm{~d}, 3 \mathrm{H},-\mathrm{CH}_{3}\right), 2.29\left(\mathrm{~s}, 3 \mathrm{H},-\mathrm{CH}_{3}\right), 2.33\left(\mathrm{~s}, 3 \mathrm{H},-\mathrm{CH}_{3}\right), 3.67(\mathrm{~s}, 3 \mathrm{H}$, $\left.-\mathrm{OCH}_{3}\right), 3.76\left(\mathrm{~s}, 3 \mathrm{H},-\mathrm{OCH}_{3}\right), 4.28(\mathrm{~m}, 1 \mathrm{H},-\mathrm{CH}), 4.40(\mathrm{~m}, 1 \mathrm{H},-\mathrm{CH}), 5.18(\mathrm{~s}, 1 \mathrm{H},-\mathrm{NH}), 6.88-$ 7.26 (m, 4H, Ar-H); MS (EI): $m / z$ 359. Anal. Calcd. for $\mathrm{C}_{20} \mathrm{H}_{25} \mathrm{NO}_{5}: \mathrm{C}, 66.83 ; \mathrm{H}, 7.01 ; \mathrm{N}, 3.90 \%$. Found: C, 66.75; H, 6.87; N, 3.85\%.

\section{General procedure for the synthesis of N-substituted 1,4-dihydropyridines (9a-d)}

A mixture of aryl aldehydes 1 (5 mmol), isopropyl acetoacetate 7 (2.93 $\mathrm{ml}, 10 \mathrm{mmol})$, aromatic amines 8 (5 mmol) was heated (without solvent) on steam bath for half an hour. After elimination of water, iodine $(0.38 \mathrm{~g}, 1.5 \mathrm{mmol})$ and ethanol $(5 \mathrm{ml})$ directly charge to the reaction mixture. The reaction mixture was then stirred at room temperature until the reaction was completed (4-5 hr monitored by TLC). The reaction mixture was treated with aq $\mathrm{Na}_{2} \mathrm{~S}_{2} \mathrm{O}_{3}$ solution, extracted into ethyl acetate $(2 \times 20 \mathrm{ml})$. The solvent was removed in vacuo and the resulting crude product $(81-84 \%)$ was recrystallized from the ethanol to give the pure compounds $9 \mathrm{a}-\mathrm{d}$. The reaction time and yields are depicted in Table 1.

Diisopropyl-1-(4-methoxyphenyl)-2,6-dimethyl-4-(3-nitrophenyl)-1,4-dihydropyridine-3,5dicarboxylate (9a). Pale yellow solid; $\mathrm{mp}$ 151-152 ${ }^{\circ} \mathrm{C}$; IR $(\mathrm{KBr}): v_{\max }=1665(-\mathrm{C}=\mathrm{O}), 1572$ $(-\mathrm{C}=\mathrm{C}), 1491,1439,1305,1235,1167,1028 \mathrm{~cm}^{-1} ;{ }^{1} \mathrm{H} \mathrm{NMR}\left(400 \mathrm{MHz}, \mathrm{CDCl}_{3}\right), \delta=1.16(\mathrm{~d}, 6 \mathrm{H}$, $\left.-\mathrm{CH}_{3}\right), 1.28\left(\mathrm{~d}, 6 \mathrm{H},-\mathrm{CH}_{3}\right), 2.08\left(\mathrm{~s}, 6 \mathrm{H},-\mathrm{CH}_{3}\right), 3.86\left(\mathrm{~s}, 3 \mathrm{H},-\mathrm{OCH}_{3}\right), 5.00(\mathrm{~m}, 2 \mathrm{H},-\mathrm{CH}), 5.14$ (s, 1H, -CH), 6.97-8.06 (m, 8H, Ar-H); ${ }^{13} \mathrm{C} \mathrm{NMR} \mathrm{(400} \mathrm{MHz,} \mathrm{CDCl}_{3}$ ), 18.77, 21.87, 22.10, 39.35, 55.56, 67.54, 105.37, 114.72, 121.32, 122.90, 128.55, 131.03, 132.65, 134.20, 148.33, 148.80, 
149.77, 159.52, 167.13; MS (EI): $m / z$ 508. Anal. Calcd. for $\mathrm{C}_{28} \mathrm{H}_{32} \mathrm{~N}_{2} \mathrm{O}_{7}: \mathrm{C}, 6.13 ; \mathrm{H}, 6.34 ; \mathrm{N}$, 5.57\%. Found: C, 66.08; H, 6.22; N, 5.45\%.

Diisopropyl-1-phenyl-2,6-dimethyl-4-(3-nitrophenyl)-1,4-dihydropyridine-3,5-dicarboxylate (9b). Pale yellow solid; mp $156-157^{\circ} \mathrm{C}$; IR (KBr): $v_{\max }=1670(-\mathrm{C}=\mathrm{O}), 1602(-\mathrm{C}=\mathrm{C})$, $1577,1483,1425,1352,1315,1240,1176,1068 \mathrm{~cm}^{-1} ;{ }^{1} \mathrm{H}$ NMR $\left(400 \mathrm{MHz}, \mathrm{CDCl}_{3}\right), \delta=1.17$ (d, $\left.6 \mathrm{H},-\mathrm{CH}_{3}\right), 1.30\left(\mathrm{~d}, 6 \mathrm{H},-\mathrm{CH}_{3}\right), 2.16\left(\mathrm{~s}, 6 \mathrm{H},-\mathrm{CH}_{3}\right), 3.82\left(\mathrm{~s}, 3 \mathrm{H},-\mathrm{OCH}_{3}\right), 5.03(\mathrm{~m}, 2 \mathrm{H},-\mathrm{CH}), 5.17$ (s, $1 \mathrm{H},-\mathrm{CH}), 7.04-8.05(\mathrm{~m}, 9 \mathrm{H}, \mathrm{Ar}-\mathrm{H})$; $\mathrm{MS}(\mathrm{EI}): \mathrm{m} / z$ 478. Anal. Calcd. for $\mathrm{C}_{27} \mathrm{H}_{30} \mathrm{~N}_{2} \mathrm{O}_{6}$ : C, 67.77; H, 6.32; N, 5.85\%. Found: C, 67.65; H, 6.28; N, 5.78\%.

Diisopropyl-1-(4-methoxyphenyl)-2,6-dimethyl-4-(4-methoxyphenyl)-1,4-dihydropyri-dine3,5-dicarboxylate (9c). Pale yellow solid; mp 148-150 ${ }^{\circ} \mathrm{C}$; IR (KBr): $v_{\max }=1664(-\mathrm{C}=\mathrm{O}), 1602$ $(-\mathrm{C}=\mathrm{C}), 1568,1448,1431,1325,1234,1174,1066 \mathrm{~cm}^{-1} ;{ }^{1} \mathrm{H}$ NMR $\left(400 \mathrm{MHz}, \mathrm{CDCl}_{3}\right), \delta=1.13$ $\left(\mathrm{d}, 6 \mathrm{H},-\mathrm{CH}_{3}\right), 1.26\left(\mathrm{~d}, 6 \mathrm{H},-\mathrm{CH}_{3}\right), 2.11\left(\mathrm{~s}, 6 \mathrm{H},-\mathrm{CH}_{3}\right), 3.73\left(\mathrm{~s}, 3 \mathrm{H},-\mathrm{OCH}_{3}\right), 3.79\left(\mathrm{~s}, 3 \mathrm{H},-\mathrm{OCH}_{3}\right)$, $5.03(\mathrm{~m}, 2 \mathrm{H},-\mathrm{CH}), 5.16(\mathrm{~s}, 1 \mathrm{H},-\mathrm{CH}), 6.95-7.93(\mathrm{~m}, 8 \mathrm{H}, \mathrm{Ar}-\mathrm{H})$; MS (EI): $\mathrm{m} / z$ 493. Anal. Calcd. for $\mathrm{C}_{29} \mathrm{H}_{35} \mathrm{NO}_{6}$ : C, 70.57; H, 7.15; N, 2.84\%. Found: C, 70.48; H, 7.09; N, 2.77\%.

Diisopropyl-1-phenyl-2,6-dimethyl-4-(4-methoxyphenyl)-1,4-dihydropyridine-3,5-dicarboxylate (9d). Pale yellow solid; $\mathrm{mp} 153-154{ }^{\circ} \mathrm{C}$; IR $(\mathrm{KBr}): v_{\max }=1662(-\mathrm{C}=\mathrm{O}), 1602(-\mathrm{C}=\mathrm{C})$, 1569, 1491, 1431, 1323, 1234, 1176, $1020 \mathrm{~cm}^{-1} ;{ }^{1} \mathrm{H}$ NMR $\left(400 \mathrm{MHz}, \mathrm{CDCl}_{3}\right), \delta=1.15(\mathrm{~d}, 6 \mathrm{H}$, $\left.-\mathrm{CH}_{3}\right), 1.33\left(\mathrm{~d}, 6 \mathrm{H},-\mathrm{CH}_{3}\right), 2.14\left(\mathrm{~s}, 6 \mathrm{H},-\mathrm{CH}_{3}\right), 3.81\left(\mathrm{~s}, 3 \mathrm{H},-\mathrm{OCH}_{3}\right), 5.07(\mathrm{~m}, 2 \mathrm{H},-\mathrm{CH}), 5.19(\mathrm{~m}$, $1 \mathrm{H},-\mathrm{CH}), 7.06-8.23$ (m, 9H, Ar-H); $\mathrm{MS}(\mathrm{EI}): \mathrm{m} / z$ 463. Anal. Calcd. for $\mathrm{C}_{28} \mathrm{H}_{33} \mathrm{NO}_{5}: \mathrm{C}, 72.55 ; \mathrm{H}$, 7.18; N, 3.02\%. Found: C, 72.45; H, 7.07; N, 2.83\%.

\section{Supplementary Information Available}

NMR and MS data.

\section{Acknowledgements}

The authors thank Department of Chemistry, Saurashtra University, Rajkot for providing facilities. We are also thankful to CIL, RSIC, Chandigarh for providing ${ }^{1} \mathrm{H}$ NMR spectral analysis and SAIF, CDRI Lucknow for elemental analysis of compounds.

\section{References}

1. Kawase, M.; Shah, A.; Gaveriya, H.; Motohashi, N.; Sakagami, H.; Varga, A.; Molnar, J. J. Bioorg. Chem. 2002, 10, 1051.

2. Suarez, M.; Verdecia, Y.; Illescas, B.; Martinez-Alvarez, R.; Avarez, A.; Ochoa, E.; Seoane, C.; Kayali, N.; Martin, N.; Tetrahedron 2003, 59, 9179. 
3. Shan, R.; Velazquez, C.; Knaus, E. E. J. Med. Chem. 2004, 47, 254.

4. Ryabokon, N.; Goncharova, R. I.; Duburs, G.; Rzeszowska-Wolny, J. Mutation Research 2005, 52, 587.

5. Yamamoto, T.; Niwa, S.; Ohno, S.; Onishi, T.; Matsueda, H.; Koganei, H.; Uneyama, H.; Fujita, S.; Takeda, T.; Kito, M.; Ono, Y.; Saitou, Y.; Takahara, A.; Iwata, S.; Shoji, M. Bioorg. Med. Chem. Lett. 2006, 16, 798.

6. Buhler, F. R.; Kiowski, W. J. Hypertens. 1987, S3, 5.

7. Reid, J. L.; Meredith, P. A.; Pasanisi, F. J. Cardiovasc. Pharmacol. 1985, S18, 7.

8. Navidpour, L.; Shafaroodi, H.; Miri, R.; Dehpour, A. R.; Shafiee, A. Il Farmaco 2004, 59, 261.

9. Budriesi, R.; Bisi, A.; Ioan, P.; Rampa, A.; Gobbi, S.; Belluti, F.; Piazzi, L.; Valenti, P.; Chiarini, A. Bioorg. Med. Chem. 2005, 13, 3423.

10. Godfraid, T.; Miller, R.; Wibo, M. Pharmacol. Rev. 1986, 38, 321.

11. Sausins, A.; Duburs, G. Heterocycles 1988, 27, 269.

12. Mager, P. P.; Coburn, R. A.; Solo, A. J.; Triggle, D. J.; Rothe, H. Drug Des. Discovery 1992, 8, 273.

13. Mannhold, R.; Jablonka, B.; Voigdt, W.; Schoenanger, K.; Schravan, K. Eur. J. Med. Chem. 1992, 27, 229.

14. Cai, X.; Yang, H.; Zhang, G. Can. J. Chem. 2005, 83, 273.

15. Hantzsch, A.; Liebigs, J. Ann. Chem. 1882, 1, 215.

16. Loev, B.; Snader, K. M. J. Org. Chem. 1965, 30, 1914.

17. Khadikar, B. M.; Gaikar, V. G.; Chitnavis, A. A. Tetrahedron Lett. 1995, 36, 8083.

18. Ohberg, L.; Westman, J. Synlett. 2001, 1296.

19. Agarwal, A.; Chauhan, P. M. S. Tetrahedron Lett. 2005, 46, 1345.

20. Ji, S.-J.; Jiang, Z.-Q.; Lu, J.; Loh, T.-P. Synlett. 2004, 831.

21. Sridhar, R.; Perumal, P. T. Tetrahedron 2005, 61, 2465.

22. Dondoni, A.; Massi, A.; Minghini, E.; Sabbatini, S.; Bertoasi,V. J. Org. Chem. 2003, 68, 6172.

23. Dondoni, A.; Massi, A.; Minghini, E.; Bertoasi, V. Tetrahedron 2004, 60, 2311.

24. Tewari, N.; Dwivedi, N.; Tripathi, R. P. Tetrahedron Lett. 2004, 45, 9011.

25. Moseley, J. D. Tetrahedron Lett. 2005, 46, 3179.

26. Sabitha, G.; Reddy, G. S. K. K.; Reddy, Ch. S.; Yadav, J. S. Tetrahedron Lett. 2003, 44, 4129.

27. Wang, L.-M.; Sheng, J.; Zhang, L.; Han, J.-W.; Fan, Z.; Tian, H.; Qian, C.-T. Tetrahedron 2005, 61, 1539.

28. Sun, J.; Dong, Y.; Wang, X.; Wang, S.; Hu, Y. J. Org. Chem. 2004, 69, 8932.

29. Phukan, P. Tetrahedron Lett. 2004, 45, 4785.

30. Ke, B.; Qin, Y.; He, Q.; Huang, Z.; Wang, F. Tetrahedron Lett. 2005, 46, 1751.

31. Banik, B. K.; Fernandez, M.; Alvarez, C. Tetrahedron Lett. 2005, 46, 2479.

32. Kartha, K. P. R. Tetrahedron Lett. 1986, 27, 3415. 
33. Jenner, G. Tetrahedron Lett. 1988, 29, 2445.

34. Bhosale, R. S.; Bhosale, S. V.; Bhosale, S. V.; Wang, T.; Zubaidha, P. K. Tetrahedron Lett. 2004, 45, 7187.

35. Ramalinga, K.; Vijayalakshmi, P.; Kaimal, T. N. B. Tetrahedron Lett. 2002, 43, 879.

36. Chavan, S. P.; Kale, R. R.; Shivasankar, K.; Chandake, S. I.; Benjamin, S. B. Synthesis 2003, 2695.

37. Hessian, K. O.; Flynn, B. L. Org. Lett. 2003, 5, 4377.

38. Ko, S.; Sastry, M. N. V.; Lin, C.; Yao, C. -F. Tetrahedron Lett. 2005, 46, 5771.

39. Zolfigol, M. A.; Saleh, P.; Khorramabadi-Zad, A.; Shayegh, M. J. Mol. Catal. 2007, 261, 88. 